\title{
Efecto del ruido del tráfico vehicular en cantos de Hylophilus flavipes y Cyclarhis gujanensis
}

\author{
Effect of the Traffic Noise on the Songs of Hylophilus Flavipes and Cyclarhis \\ gujanensis
}

\author{
G. Pacheco-Vargas ${ }^{\mathrm{a}, *}$ \\ S. Losada-Prado ${ }^{\text {a }}$
}

Recepción: 13-abr-15

Aceptación: 17-jul-15

\begin{abstract}
Resumen
La hipótesis de adaptación acústica ha justificado que la estructura del hábitat y la intervención antrópica son factores que influyen en la variación de las vocalizaciones de las aves. Este estudio estimó el efecto del ruido del tráfico vehicular sobre las vocalizaciones de Hylophilus flavipes y Cyclarhis gujanensis. Se efectuaron transectos paralelos a carreteras en dos localidades del bosque seco tropical del departamento del Tolima; se realizó la grabación de sus cantos y la medición de la intensidad de ruido emitido por los vehículos. Se encontró que $H$. flavipes y $C$. gujanensis emiten cantos con una frecuencia mínima mayor cuando se encuentran en lugares donde la intensidad del ruido está por encima de los $40 \mathrm{~dB}$. Así mismo, $H$. flavipes genera cantos con una menor duración de notas y una mayor duración entre notas, y tiende a cantar con menor número de notas cuando se ubican cerca de la carretera. Además, la última nota de los cantos de $C$. gujanensis tiende a ser más duradera bajo estas condiciones. Este estudio permitió determinar una variación en las vocalizaciones de las dos especies ante este factor, evidenciándose una respuesta mayor en H. flavipes que en C. gujanensis.
\end{abstract}

Palabras clave: canto, Cyclarhis gujanensis, Hylophilus flavipes, ruido, tráfico vehicular.

\begin{abstract}
The acoustic adaptation hypothesis had evidenced that the structure of habitat and human intervention are factors that influence the variation in bird vocalizations. This study estimated the effect of road traffic noise on the vocalizations of Cyclarhis gujanensis and Hylophilus flavipes. Parallel transects were laid at two locations in tropical dry forest in the Tolima department, and the songs and noise intensity measurements were recorded. H. flavipes and C. gujanensis sang songs with a higher minimum frequency when in places with noise level above $40 \mathrm{~dB}$. Additionaly, H. flavipes sang songs with a shorter duration of notes and longer duration between notes, and sang with fewer notes in places close to the highway. Furthermore, the last note of $C$. gujanensis songs tended to be longer under these conditions. This study demonstrated a variation in vocalizations of two species under road traffic noise, with a greater response from $H$. flavipes than $C$. gujanensis.
\end{abstract}

Key words: Noise, Road traffic noise, Rufous-browed Peppershrike, Scrub greenlet, Song.

${ }^{a}$ Grupo de Investigación en Zoología, Facultad de Ciencias, Universidad del Tolima.

*Autor de correspondencia: gfpacheco@ut.edu.co 


\section{Introducción}

El canto de un ave es una señal utilizada en la comunicación con individuos de la misma especie, o de especies distintas, buscando modificar el comportamiento del animal que la recibe. Entre las funciones del canto se han propuesto el reconocimiento, la defensa del territorio y la selección sexual [1]. De otro lado, desde que se planteó la hipótesis de adaptación acústica [2], se han venido estudiando los aspectos que influyen en la variación del canto en las aves y se ha encontrado que la estructura del hábitat y, más recientemente, la intervención antrópica [3-6] son factores que influyen en la variación de las vocalizaciones.

De esta manera, el ruido que provocan diferentes actividades humanas interfiere en la comunicación acústica de las aves, causando error en la comunicación [7]. Como respuesta, algunas especies aumentan la frecuencia del canto, y se ha encontrado que este incremento ocurre en mayor proporción en las zonas urbanas, en comparación con las zonas periurbanas y rurales [5-12]. Esto ha sido documentado en las siguientes especies: Trichoglossus haematodus (Rainbow lorikeet) [8], Platycercus eximius (Eastern rosella)[8], Anthochaera carunculata (Red wattlebird) [8], Manorina melanophrys (Bell miner) [8], Cracticus torquatus (Grey butcherbird) [8], Troglodytes musculus (Southern House Wren) [9], T. troglodytes (Eurasian wren)[11], Turdus merula (Common blackbird) [8, 10], Parus major (Great tit) [5], Melospiza melodía (Song sparrow)[12]. Así, el objetivo de este estudio fue determinar el efecto del ruido producido por el tráfico vehicular, en vías primarias, sobre las vocalizaciones de las especies de aves Cyclarhis gujanensis e Hylophilus flavipes en la zona de vida bosque seco tropical en el departamento del Tolima.

\section{Metodología}

El estudio se llevó a cabo en dos localidades: el Centro Universitario Regional del Norte (CURDN) de la Universidad del Tolima (N 0500'3,5"; W $74^{\circ} 28^{\prime} 32^{\prime \prime}$ a $280 \mathrm{msnm}$ ), en el municipio de ArmeroGuayabal, por donde pasa la vía que lleva hacia el norte del Tolima, y la vereda Potrerillo, entre los municipios de Coello y El Espinal, por donde cruza la vía que conduce hacia la ciudad de Bogotá D.C. (N 04¹4'55,30"; W 74 58'40,60" a 430 msnm). La distancia entre estas dos localidades es de $85 \mathrm{~km}$, aproximadamente.

Las especies $C$. gujanensis e H. flavipes se encuentran reportadas dentro de la avifauna de esta zona de vida en el departamento del Tolima [13]. La fase de campo se desarrolló entre marzo y agosto de 2013; se realizaron tres muestreos en cada una de las dos localidades. Se diseñaron tres transectos de $2 \mathrm{~km}$ de longitud, paralelos a la carretera (figura 1). El primero (T1) en una franja de 0 a $160 \mathrm{~m}$; el segundo (T2), de 160 a $900 \mathrm{~m}$, y el tercero (T3), a más de $900 \mathrm{~m}$; teniendo en cuenta como referencia la carretera. En cada transecto se midió la intensidad del ruido producido por el tráfico vehicular, usando un sonómetro Extech 407730. La grabación de los cantos de $C$. gujanensis e H. flavipes se realizó con el uso de un equipo de bioacústica compuesto por una grabadora digital Marantz PMD 661, un micrófono unidireccional Sennheiser ME 62 y una antena parabólica Sennheiser. A partir de las mediciones realizadas a la intensidad de ruido generado por el tráfico vehicular en las dos localidades, se establecieron dos tratamientos: el primero con intensidad $>40$ $\mathrm{dB}$, el cual comprendió el transecto $\mathrm{T} 1$, y el segundo con intensidad $<40 \mathrm{~dB}$, el cual comprendió los transectos T2 y T3 (figura 1). Todos los cantos fueron registrados entre las 06:00 y las 11:00 horas, en recorridos paralelos a las carreteras, lo que permitió una secuencialidad en las grabaciones.

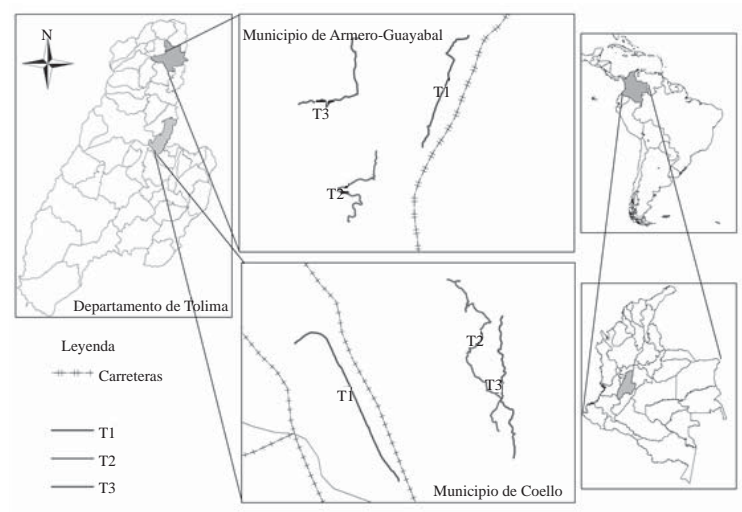

Figura 1. Mapa de los transectos en las localidades del bosque seco tropical del departamento del Tolima. Municipio de Armero-Guayabal (CURDN), Municipio de Coello (Potrerillo). T1=>40 dB, T2 y T3 $=<40 \mathrm{~dB}$.

La independencia en los cantos analizados se logró ubicando los sitios de los individuos vocalizantes, lo cual garantizó que los cantos procesados provenían de individuos diferentes. Los recortes de 
las grabaciones de las aves registradas en campo se efectuaron con el programa Audacity ${ }^{\circledR}$ 2.0.0 [14]. Los espectrogramas fueron generados con el programa SYRINX-PC ${ }^{\circledR}$ [15], configurando un tamaño de transformación de 1024 y seleccionando una ventana tipo Blackman en la transformación rápida de Fourier. De esta forma, se analizaron 71 cantos de $H$. flavipes y 68 de $C$. gujanensis. Las variables bioacústicas analizadas fueron: frecuencia mínima (fmin), frecuencia máxima (fmax), rango de frecuencia (ranfrec), duración de la nota (durnota), duración entre notas (internota), duración total del canto (durtot) y número de notas (numnot) (figura 2). Además, en $H$. flavipes se analizaron todas las notas de cada canto, debido a que esta especie solo posee un canto, que guarda siempre el mismo patrón, consistenteen una nota modulada que aumenta de frecuencia a través del tiempo en un rango de $2-4 \mathrm{kHz}$, la cual es emitida rápida y constantemente hasta más de 20 veces (figura $2 \mathrm{~b}$ ). En cuanto a $C$. gujanensis, se determinó que posee cinco cantos distintos, que tienen de tres a siete notas; por lo tanto, se tuvo que buscar un patrón en el canto que permitiera ser comparado, y se estableció que la última nota de todos los cantos siempre guarda el mismo patrón (forma de letra M y mayor duración); de esta manera, los análisis involucraron las variables anteriormente mencionadas, y la última nota del canto de esta especie. Además, se analizó la duración total del canto (durtot) y el número de notas (numnot), pero se eliminó del análisis la variable duración entre notas (internota) (figura 2a).

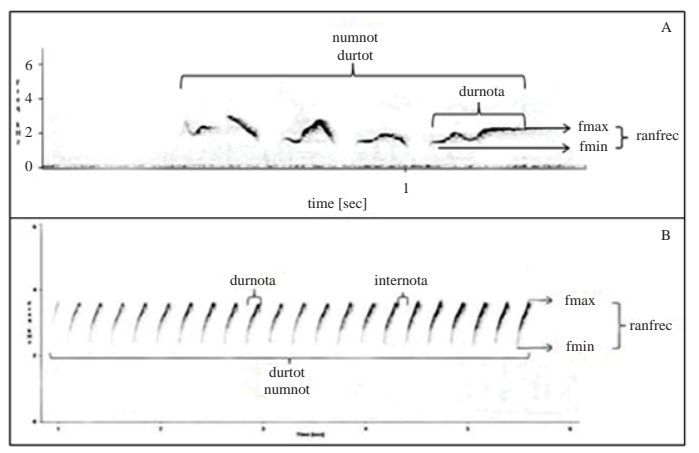

Figura 2. Variables bioacústicas. A. C. gujanensis. B. H. flavipes.

Las variables bioacústicas fueron analizadas mediante estadística descriptiva, usando gráficas boxplot con ayuda del programa InfoStat versión $2012^{\circledR}$ [16]; para determinar diferencias significativas entre tratamientos para las variables evaluadas en los cantos se realizaron pruebas $t$ de Student $(\alpha=0,05)$ utilizando el programa STATISTICA $8^{\circledR}$ [17]. Todas las variables fueron sometidas a las pruebas de normalidad y homogeneidad de varianzas.

\section{Resultados y discusión}

Los cantos de H. flavipes, en el tratamiento con intensidad de ruido $>40 \mathrm{~dB}$, muestran los mayores valores de frecuencia mínima, frecuencia máxima y duración entre notas. En este tratamiento es menor el rango de frecuencia, la duración de la nota y el número de notas. La duración del canto se mantiene estable en los dos tratamientos (figura 3). Se presentaron diferencias significativas $(p<0,05)$ en la frecuencia mínima, el rango de frecuencia, la duración de las notas y la duración entre notas entre tratamientos (tabla 1).

En cuanto a $C$. gujanensis, la frecuencia mínima y la duración de la última nota son mayores en el tratamiento con intensidad de ruido $>40 \mathrm{~dB}$; en este tratamiento, el número de notas es menor. La frecuencia máxima, el rango de frecuencia y la duración del canto se mantienen estables en los dos tratamientos (figura 4). Existen diferencias significativas $(p<0,05)$ en la frecuencia mínima al comparar los cantos en los dos tratamientos (tabla 2).

La polución sonora de origen antropogénico provoca que las aves modifiquen el canto para evitar el efecto de enmascaramiento [18]. En este estudio se encontró que los individuos de $H$. flavipes y $C$. gujanensis emiten cantos con una frecuencia mínima mayor cuando se encuentran en lugares cercanos a la carretera, donde la intensidad de ruido producido por los vehículos que transitan es $>40 \mathrm{~dB}$. Este hecho se ha registrado en diferentes especies de aves: Collurincincla harmónica (Australia) y Parus major y Turdus merula (Europa), las cuales aumentan la frecuencia del canto para evitar el enmascaramiento provocado por el ruido proveniente del tráfico que circula por las carreteras [19, 20, 21, 22]. Mendoza y Arce-Plata [23] encontraron que Pitangus sulphuratus emitía cantos con frecuencias más altas en las dos primeras notas cuando los individuos estaban presentes en zonas urbanas de Santiago de Cali (Colombia). Francis et ál. [24] hallaron que Pipilo maculatus cantaba a una frecuencia mayor cuando se expone al ruido del compresor de un pozo de gas natural en San Juan (Estados Unidos). 


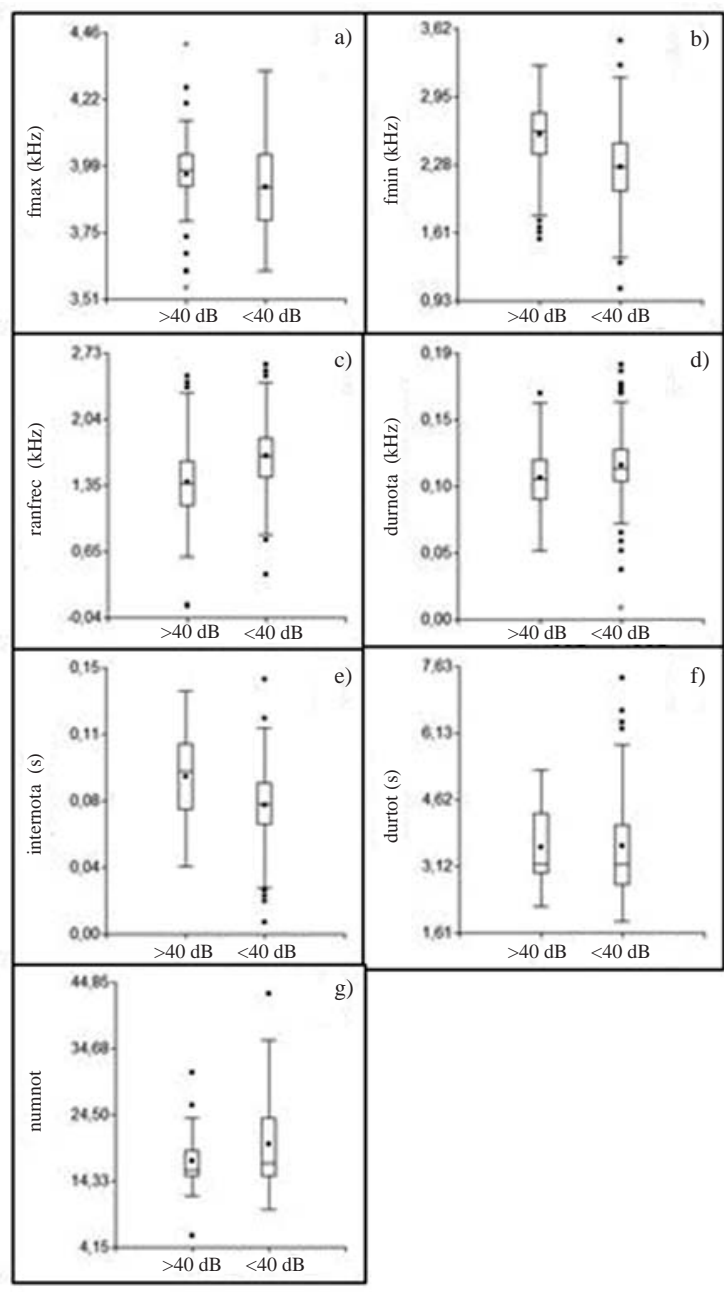

Figura 3. Gráficas box-plot para las variables bioacústicas del canto de $H$. flavipes. a) frecuencia máxima; b) frecuencia mínima; c) rango de frecuencia; d) duración de nota; e) duración entre notas; f) duración del canto; g) número de notas.

La tendencia de aumentar la frecuencia del canto en las aves en respuesta al ruido se debe al solapamiento entre la frecuencia del canto y la frecuencia del ruido [22, 25]; esto se explica porque, según Dooling y Popper [26], cuando un vehículo en movimiento produce una intensidad de ruido $>40 \mathrm{~dB}$, se da como consecuencia el enmascaramiento del canto en las aves; en las mediciones se obtuvieron valores promedio de $69,34 \mathrm{~dB}$ y $55,31 \mathrm{~dB}$, para Potrerillo y CURDN, respectivamente, lo que quiere decir que en las localidades evaluadas el sonido de los autos logra enmascarar los cantos de las especies de aves estudiadas a una distancia de $160 \mathrm{~m}$ de la vía principal. Por lo anterior, los individuos de las especies H. flavipes y C. gujanensis aumentan la frecuencia
Tabla 1. Valores de la prueba $t$ de Student para $H$. flavipes. (n: número de nota). ${ }^{* *}$ Diferencia significativa $(p<0,05)$.

\begin{tabular}{lccc}
\hline Variable & $t$ & $\mathrm{gl}$ & $p$ \\
\hline n1fmin & 3,87616 & 68 & $0,000242^{* *}$ \\
n1ranfrec & $-3,26963$ & 68 & $0,001692^{* *}$ \\
n1durnot & $-2,31225$ & 68 & $0,023798^{* *}$ \\
n2fmin & 3,60837 & 68 & $0,000584^{* *}$ \\
n2ranfrec & $-3,07669$ & 68 & $0,003015^{* *}$ \\
n3fmin & 2,86495 & 68 & $0,005544^{* *}$ \\
n3ranfrec & $-2,27954$ & 68 & $0,025777^{* *}$ \\
n3durnot & $-3,01537$ & 68 & $0,003607^{* *}$ \\
n4fmin & 2,83774 & 68 & $0,005983^{* *}$ \\
n4ranfrec & $-2,19739$ & 68 & $0,031403^{* *}$ \\
n4durnot & $-2,88391$ & 68 & $0,005255^{* *}$ \\
n5fmin & 2,81732 & 68 & $0,006334^{* *}$ \\
n5ranfrec & $-2,17051$ & 68 & $0,033464^{* *}$ \\
n5durnot & $-2,15466$ & 68 & $0,034734^{* *}$ \\
n6fmin & 2,30841 & 68 & $0,024023^{* *}$ \\
n7fmin & 2,28894 & 67 & $0,025242^{* *}$ \\
n8fmin & 2,30131 & 67 & $0,024492^{* *}$ \\
n9fmin & 2,39702 & 67 & $0,019325^{* *}$ \\
n9ranfrec & $-2,03942$ & 67 & $0,045355^{* *}$ \\
n10fmin & 2,29535 & 67 & $0,024851^{* *}$ \\
n26durnot & 4,43799 & 9 & $0,001628^{* *}$ \\
n27durnot & 3,14529 & 7 & $0,016259^{* *}$ \\
dur tot & $-1,26998$ & 68 & 0,208422 \\
num not & $-1,56406$ & 68 & 0,122445 \\
internota 1 & 4,74759 & 68 & $0,000011^{* *}$ \\
internota 2 & 3,41051 & 68 & $0,001095^{* *}$ \\
internota 3 & 4,32773 & 68 & $0,000051^{* *}$ \\
internota 5 & 3,72468 & 68 & $0,000400^{* *}$ \\
internota 7 & 2,70856 & 67 & $0,008569^{* *}$ \\
internota 8 & 3,38033 & 67 & $0,001211^{* *}$ \\
internota 9 & 2,98068 & 67 & $0,004005^{* *}$ \\
internota 10 & 2,76296 & 66 & $0,007414^{* *}$ \\
internota 11 & 2,96750 & 65 & $0,004199^{* *}$ \\
internota 12 & 2,19654 & 60 & $0,031928^{* *}$ \\
internota 13 & 2,42049 & 57 & $0,018709^{* *}$ \\
internota 14 & 2,46124 & 53 & $0,017135^{* *}$ \\
internota 15 & 2,15425 & 45 & $0,036612^{* *}$ \\
\hline & & &
\end{tabular}

Tabla 2. Valores de la prueba $t$ de Student para C. gujanensis.(n: número de nota). **Diferencia significativa $(p<0,05)$.

\begin{tabular}{lccc}
\hline Variable & $t$ & gl & $p$ \\
\hline fmax & 0,04916 & 68 & 0,960936 \\
fmin & 2,06363 & 68 & $0,042873^{* *}$ \\
ranfrec & $-0,77922$ & 68 & 0,438556 \\
durnota & 1,27641 & 68 & 0,206155 \\
durtot & 0,03107 & 68 & 0,975303 \\
numnot & $-1,16815$ & 68 & 0,246825 \\
\hline
\end{tabular}

mínima del canto para evitar dicho efecto, logrando así evitar el solapamiento de la frecuencia más baja de los cantos con el ruido. Además, como consecuencia del aumento en la frecuencia mínima, el 


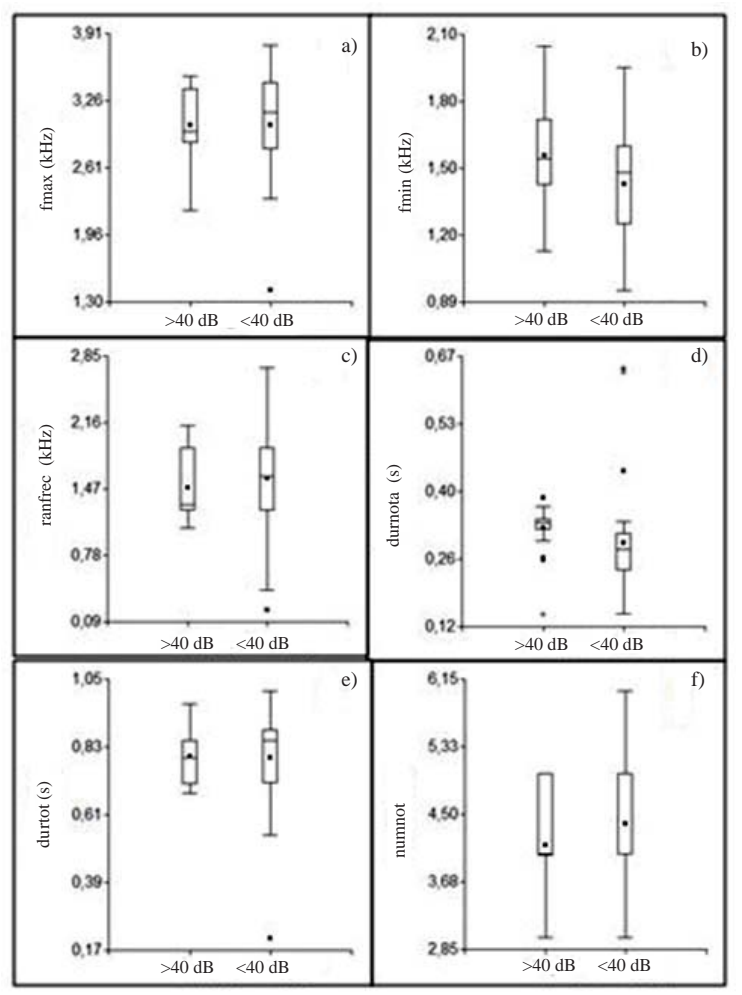

Figura 4. Gráficas box-plot para las variables bioacústicas del canto de $C$. gujanensis evaluadas en los dos tratamientos para la localidad del CURDN. a) frecuencia máxima; b) frecuencia mínima; c) rango de frecuencia; d) duración de nota; e) duración del canto; f) número de notas.

rango de frecuencia se reduce en los cantos emitidos por $H$. flavipes a pesar de que hay una tendencia a aumentar la frecuencia máxima. Sin embargo, esto no es suficiente para que se mantenga constante el rango de frecuencia en los dos tratamientos.

En cuanto a las variables temporales del canto, hay que destacar que los individuos de $H$. flavipes generan cantos con menor duración de notas, y tienden a cantar con menor número de notas cuando se ubican en lugares donde la intensidad del ruido es $>40 \mathrm{~dB}$, es decir, a una distancia inferior a los 160 $\mathrm{m}$. Esto puede ser debido a que cualquier variación o aumento en las frecuencias, aunque pequeño, supone un posible gasto de energía relacionado con el consumo de oxígeno y el aumento de la intensidad vocal [27, 28]. Para este caso, en las áreas donde se percibe ruido los individuos se ven obligados a incrementar la frecuencia del canto; por lo tanto, la energía que necesitan para efectuar esta actividad podría ser mayor, lo que no les permitiría emitir notas largas, ni un mayor número de notas. Además, se puede afirmar en este trabajo que $H$. flavipes canta con una duración entre notas mayor al encontrarse en lugares de alta intensidad de ruido producido por vehículos; una condición similar no ha sido reportada.

Por último, en cuanto a las variables temporales del canto, vale la pena rescatar que en el presente estudio se encontró que la última nota de los cantos de $C$. gujanensis tiende a ser más duradera cuando los individuos se encuentran en un área donde la intensidad de ruido es $>40 \mathrm{~dB}$; algo similar fue registrado por Mendes et ál. [9], al comparar la relación del canto de Troglodytes musculus con el ruido de las áreas urbanas, periurbanas y rurales en Belém do Pará (Brasil); sin embargo, este autor afirmó que dicho efecto se debía a las diferencias en la estructura de los tres hábitats, pero los transectos realizados en este estudio se llevaron a cabo en coberturas vegetales muy similares (crecimiento secundario); por lo tanto, tal factor no determina esta respuesta. La mayor duración de esta nota podría relacionarse como un efecto del ruido.

\section{Conclusión}

Este estudio permitió evidenciar una variación en las vocalizaciones de las dos especies a causa del ruido producido por el tráfico vehicular; mayor en $H$. flavipes que en $C$. gujanensis. Se recomienda evaluar el efecto del ruido sobre las vocalizaciones de otras especies de aves canoras. No fue posible marcar individuos y conocer si se movían entre tratamientos; sin embargo, se considera que debe avaluarse la territorialidad de la especie en estos tratamientos.

\section{Agradecimientos}

A la Oficina de Investigaciones y Desarrollo Científico de la Universidad del Tolima, por la financiación de esta investigación. Esta es la contribución \#475 del Grupo de Investigación en Zoología de la Universidad del Tolima.

\section{Referencias}

[1] C. K. Catchpole, and P. J. B. Slater, Bird song: Biological themes and variations, Cambridge University Press, Cambridge, United Kingdom, 1995.

[2] P. Hansen, "Vocal learning: its role in adapting sound structures to long-distance propagation 
and a hypothesis on its evolution", Animal Behaviour, vol. 27, pp. 1270-1271, 1979.

[3] R. H. Wiley, "Associations of song properties with habitats for territorial oscine birds of eastern North America", American Naturalist, vol. 138, pp. 973-993, October, 1991.

[4] A. V. Badyaev, and E. S. Leaf, "Habitat associations of song characteristics in Phylloscopus and Hippolais warblers", The Auk, vol. 114, No. 1, pp. 40-46, January 1997.

[5] H. Slabbekoorn, "Songs of the city: noisedependent spectral plasticity in the acoustic phenotype of urban birds", Animal Behaviour, pp. 1-11, 2013.

[6] H. Brumm, "The impact of environmental noise on song amplitude in a territorial bird", Journal of Animal Ecology, vol. 73, no. 3, pp. 434440, May, 2004.

[7] R. J. Dooling, and H. S. H. Blumenrath, "Avian Sound Perception in Noise", Animal communication and nois. H. Brumm, Springer-Verlag, Berlin, Germany, 2013, pp. 229-251.

[8] Y. Hu, and G. C. Cardoso, "Which birds adjust the frequency of vocalizations in urban noise?", Animal Behaviour, vol. 79, pp. 863-867, April, 2010.

[9] S. Mendes, V. J. Colino-Rabanal, y S. Peris, "Diferencias en el canto de la ratona común (Troglodytes musculus) en ambientes con distintos niveles de influencia humana", Hornero, vol. 26, no. 2, pp. 85-93, diciembre 2011.

[10] S. Mendes, V. J. Colino-Rabanal, and S. Peris, "Bird song variations along an urban gradient: The case of the European blackbird (Turdus merula)", Landscape and Urban Planning, vol. 99, pp. 51-57. January 2011.

[11] S. Mendes, K. V. Cavalcante, V. J. ColinoRabanal, y S. J. Peris, "Evaluación del impacto de la Contaminación Acústica en el rango de vocalización de Paseriformes basado en el SILSpeech Interference Level", Revista de Acústica, vol. 41, pp. 33-41, 2010.

[12] W. E. Wood, and S. M. Yezerinac, "Song sparrow (Melospiza melodia) song varies with urban noise", The Auk, vol. 123, no. 3, pp. 650659, July 2006.
[13] S. Losada-Prado, y Y. G. Molina-Martínez, "Avifauna del bosque seco tropical en el departamento del Tolima (Colombia): Análisis de la comunidad", Caldasia, vol. 33, pp. 271-294, 2011.

[14] D. Mazzoni, Audacity ${ }^{\circledR}$ 2.0.0. Audacity Team, Boston, 2012.

[15] J. Burt, SYRINX-PC Version 2.6f. 2006.

[16] J. A. Di Rienzo, F. Casanoves, M. G. Balzarini, L. González, M. tablada, y C. W. Robledo, InfoStat versión 2012. Grupo InfoStat, FCA, Universidad Nacional de Córdoba, Argentina, 2012. http://www.infostat.com.ar.

[17] StatSoft, Inc. STATISTICA (data analysis software system), version 8.0, 2007. www.statsoft.com.

[18] C. P. Ortega, "Effects of noise pollution on birds: A brief review or our knowledge", Ornithological Monographs, vol. 74, pp. 6-22, 2012.

[19] F. E. Rheindt, "The impact of roads on birds: Does song frequency play a role in determining susceptibility to noise pollution?", Journal für Ornithologic, vol. 144, pp. 295-306. 2003.

[20] M. Katti, and P. S. Warren, "Tits, noise and urban bioacoustics", Trends in Ecology and Evolution, vol. 19, no. 3, pp. 109-111, March, 2004.

[21] K. M. Parris, and A. Scheneider, "Impacts of traffic noise and traffic volume on birds of roadside habitats", Ecology and Society, vol.14, no. 1, pp. 29, 2009.

[22] W. Halfwerk, L. J. Holleman, C. M. Lessells, and H. Slabbekoorn, "Negative impact of traffic noise on avian reproductive success", Journal of Applied Ecology, vol. 48, pp. 210-219, February, 2011.

[23] A. M. Mendoza, y M. I. Arce-Plata, "Aproximación al impacto de la perturbación urbana en las vocalizaciones de Pitangus sulphuratus (Tyrannidae) en Santiago de Cali, Valle del Cauca (Colombia)", Revista de Ciencias, vol. 16, pp. 19-29, 2012.

[24] C. D. Francis, N. J. Kleist, B. J. Davidson, C. P. Ortega, and A. Cruz, "Behavioral responses by two songbirds to natural-gas-well compressor noise", Ornithological Monographs, vol. 74, pp. 36-46, July 2012. 
[25] H. Slabbekoorn, and M. Peet, "Birds sing at a higher pitch in urban noise", Nature, vol. 424, pp. 267, July 2003.

[26] R. J. Dooling, and A. N. Popper, The effects of highway noise on birds, University of Maryland Press. Maryland, 2007.

[27] K. Oberweger, and F. Goller, "The metabolic cost of birdsong production", Journal of
Experimental Biology, vol. 204, pp. 3379-3388, October 2001.

[28] H. Brumm, and D. Todt, "Noise-dependent song amplitude regulation in a territorial songbird", Animal Behaviour, vol. 63, pp. 891-897, May 2002. 